Military Technical College Kobry El-Kobba, Cairo, Egypt

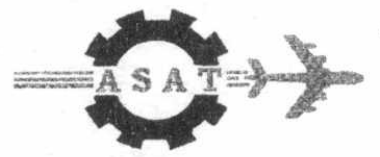

11-th International Conference on Aerospace Sciences \& Aviation Technology

\title{
AUTONOMOUS UAV FUZZY LOGIC NAVIGATION AND OBSTACLE AVOIDANCE CONTROLLER
}

\author{
AMR* A. H., SERAG" E.-D. H., MOHAMED H."
}

\begin{abstract}
Unmanned Aerial Vehicles (UAVs) are defined by the United States Department of Defense (DOD) as powered, aerial vehicles that do not carry a human operator. UAVs control systems have assumed an increasingly important role in the development and advancement of modern civilization and technology. In recent years, intelligent control of autonomous navigation of UAVs has gained much interest mainly: neural networks, non-linear adaptive control, fuzzy logic as well as combinations of these methods, such as, neuro-fuzzy control, or fuzzy logic and evolutionary or genetic algorithms. The purpose of this paper is to develop an autonomous UAV fuzzy Logic navigation and obstacle avoidance controller that is capable to perform a way point navigation and obstacles avoidance along its flight. The navigation and obstacle avoidance behavior is transformed via fuzzy rules. Multiplatform simulation package is developed to perform closed loop on-line environment sensing. Several simulation tests have been conducted on the new controller. These tests show that the proposed controller achieves the desired performance with respect to its sensors capability and limitations.
\end{abstract}

\section{KEY WORDS}

AUTONOMOUS UAV, Fuzzy Logic, Obstacle Avoidance, and Control. 


\section{INTRODUCTION}

Unmanned Aerial Vehicles (UAVs) are defined by the United States Department of Defense (DOD) as powered, aerial vehicles that do not carry a human operator [1]. These UAVs use aerodynamic forces to provide vehicle lift. They can fly in a desired non-ballistic path (user defined path) autonomously or can be piloted remotely. They can be expendable or recoverable. They carry cameras, sensors, communication equipments or other non-lethal or lethal payloads. With over 200 UAV types currently in use throughout the world [2], UAVs now have a variety of military and commercial uses. Intelligent control in the field of autonomous navigation of UAVs has been developed intensely in the last decade [3]. More and more researches are occupied with the development of intelligent navigation controllers and autopilots in order to face this attractive problem. Some of the methodological frameworks employed are, neural networks [4], non-linear adaptive control [5], fuzzy logic [6] as well as combinations of these methods, such as, neuro-fuzzy control, or fuzzy logic and evolutionary or genetic algorithms [7].

In this paper, we present a new intelligent approach for developing an autonomous UAV that is capable of performing way point navigation. Moreover it can perform obstacle avoidance along its flight. The rest of this paper is organized in the following manner: In section 2 the Beaver model is briefly presented. This model is used as a mathematical model for the development of the new controller. Design of new fuzzy logic controller is presented in section 3 . In section 4 system integration is explained while multi-platform description is presented in section 5 . Section 6 presents several tests of the new fuzzy logic controller. Finally, in section 7 we present conclusions and future extensions of this work.

\section{BEAVER MODEL}

The aircraft model which will be used in our work is "DeHavilland DHC-2 'Beaver"[8]. The Beaver, shown in Fig. 1, was designed and built in response to the demands of Canadian bush operators. Beaver was a robust aircraft with excellent short take-off-and-landing capability even with heavy loads. The main characteristics of the Beaver are shown in Table 1. The mathematical model of this aircraft is available as a MATLAB/SimuLink [9] add-in Toolbox. This module is called Flight Dynamics and Control (FDC). It is a graphical software environment for the design and analysis of aircraft dynamics and control systems. The MATLAB/SimuLink Beaver Model is shown in Fig. 2. This model is an open-loop user interactive model and allows it to be controlled as if a human pilot is onboard. This model have been tested and verified to ensure its results.

\section{FUZZY LOGIC CONTROLLER DESIGN}

Our objective in this section is to explain the development of a New Beaver Fuzzy Logic controller. This controller allows the Beaver to navigate through pre-specified way points and generate real time decisions to avoid obstacles along its flight. In order to fulfill our objective we will mention some assumptions: 
- Three Laser Range Finders (LRFs) are mounted on the Beaver. These (LRFs) can measure the distance up to $2200 \mathrm{~m}$ with accuracy $\pm 2 \mathrm{~m}$. The layout of these sensors is shown in Fig. 3.

- The new controller must supply the Beaver model with all necessary input signals needed during the flight and maintain the aircraft stability.

- The new controller must maintain constant aircraft altitude during any maneuver along the flight.

- The new controller must support 2 modes of operation (autonomous mode and remote piloted mode).

- The new controller must reach each way point with a circle of acceptance equal to 500 meter.

The basic purpose of the navigation system is the motion of the vehicle upon a predefined trajectory. In order to navigate the aircraft upon a trajectory, a fuzzy controller of the so-called Mamdani type, has been developed. Furthermore, the obstacle avoidance system is responsible for the aircraft safety against existing obstacles in unknown terrain. Both systems are merged together into the single controller as shown in Fig. 4.

The new fuzzy controller takes as inputs the sensors readings, obstacle indicator, normalized range to next waypoint, and azimuth to the next waypoint. The output of the new controller is the control surfaces deflection, the èngine rpm and the manifold pressure.

\subsection{Inputs for Fuzzy Logic Control System}

The input linguistic variables are S 1 (Forward), S2 (Right), S3 (Left), Obstacle Flag, Range, Azimuth\}. The first three linguistic variables have the same Linguistic values. The Linguistic values representing the, linguistic variables S1 (Forward), S2 (Right), S3 (Left) are: $\{$ Near, Far, Very Far, Free\}. The input membership function is shown in Fig. 5. The Obstacle Flag membership function is shown in Fig. 6. Linguistic values representing the linguistic variable Obstacle Flag are: $\{$ No Obstacle, Obstacle\}. The normalized range membership function is shown in Fig. 7. Linguistic values representing the linguistic variable Range are: \{Near, Med, Far\}. The azimuth membership function is shown in Fig. 8. Linguistic values representing the linguistic variable Azimuth are: $\{$ Left, Ahead, Right\}.

\subsection{Outputs for Fuzzy Logic Control System}

The fuzzy controller has six outputs, which are the control surfaces deflection $\left(\delta_{e}, \delta_{a}, \delta_{r}\right.$ and $\left.\delta_{f}\right)$, the engine rpm $(\mathrm{N})$, and the manifold pressure $(\mathrm{Pz})$. Similar to the inputs, the $\delta_{\mathrm{e}}$ (Elevator deflection) membership function is shown in Fig. 9. Linguistic values representing the linguistic variable $\delta_{e}$ are: $\{$ Hard Climb, Medium Climp, Soft Climb, Normal, Soft Descent, Medium Descent, Hard Descent\}. The $\delta_{a}$ (Aileron deflection) membership function is shown in Fig. 10. Linguistic values representing the linguistic variable $\delta_{a}$ are: $\{$ Hard Right Bank, Medium Right Bank, Soft Right Bank, Normal, Soft Left Bank, Medium Left Bank, Hard Left Bank\}. The $\delta_{r}$ (Rudder deflection) membership function is shown in Fig. 11. Linguistic values representing 
the linguistic variable $\delta_{\mathrm{r}}$ are: $\{$ Hard Right, Medium Right, Soft Right, Normal, Soft Left, Medium Left, Hard Left\}. The $\delta_{f}$ (Flaps deflection) membership function is shown in Fig. 12. Linguistic values representing the linguistic variable $\delta_{f}$ are: $\{$ Hard Climb, Medium Climb, Soft Climb, Normal, Soft Descent, Medium Descent, Hard Descent\}. The $N$ (Engine RPM) membership function is shown in Fig. 13. Linguistic values representing the linguistic variable $\mathrm{N}$ are: \{Cruise Descent, Climb Approach, and Take Off\}. The $\mathrm{Pz}$ (manifold pressure) membership function is shown in Fig. 14. Linguistic values representing the linguistic variable $\mathrm{Pz}$ are: \{Descent Approach, Cruise, Climb, and Take Off\}.

\subsection{The rule base}

The controller has been developed using 42 IF-THEN rules. An example of navigation rules is demonstrated: If (Range is Near) and (Azimuth is Left) Then (סe is Hard Climb) ( $\delta a$ is Hard Left Bank) ( $\delta r$ is Soft Left) ( $\delta f$ is Normal) ( $N$ is Cruise Descent) ( $\mathrm{Pz}$ is Cruise). Another example for obstacle avoidance rules is also demonstrated: If (S1 (Forward) is Free) and (S2 (Right) is Free) and (S3 (Left) is Near) then ( $\delta e$ is Medium Climb) ( $\delta a$ is Hard Right Bank) ( $\delta r$ is Soft Right) ( $\delta f$ is Normal) ( $\mathrm{N}$ is Cruise) ( $\mathrm{Pz}$ is Cruise). The development of these rules is based on consecutive tests and their control output surface is shown in Fig. 15.

\section{SYSTEM INTEGRATION}

After integrating both controllers, the Beaver model is modified as shown in Fig. 16 . This modified model has 6 inputs (Laser range finder (LRF) reading, the range and azimuth error signal to next way point) and 16 outputs (state variables that tell about the aircraft position, orientation, speed...etc). Also these modifications allow two modes of operation (autonomous/remotely piloted mode). Fig.17 shows a flow chart depicting the overall sequence of operation of our UAV controller. For each state of the UAV (position and velocity) the system acquires the LRFs data. Consequently, it decides which fuzzy rule set, the navigation set or the obstacle avoidance set, applies. Next the fuzzy controller outputs are evaluated and exported to the UAV model. The time is incremented and the state of the UAV is updated.

\section{MULTI-PLATFORM DESCRIPTIN}

The previous MATLAB/SIMULINK model is capable of simulating discrete event situations, (i.e. give the Fuzzy controller certain instant input and notice the behavior of the Beaver via the output state variable) and this is insufficient to ensure the reliability of the controller and the stability of the Beaver. Therefore, a software simulation package has been developed. This package - which is called "AeroLink"gives us the ability to do the following:

- Plot a set of waypoints for the Beaver to follow before or during its flight.

- Control the Beaver controller operation mode (remote / autonomous).

- Monitor the Beaver model state variables values.

- Monitor the Beaver maneuvers graphically.

- Control the MATLAB/SIMULINK simulation time.

- Loading multiple terrains with various obstacles. 
- Monitor the range and azimuth errors with the next waypoints.

- Monitor the controller decisions graphically.

- Monitor the Beaver trajectory along its flight.

This software, package"AeroLink" has been developed using Microsoft Visual C++. AeroLink is, a real time graphical package that uses all the power of MATLAB/SIMULINK as a hidden mathematical engine. AeroLink is used to perform closed loop continuous simulation. AeroLink trigger the MATLAB/SIMULINK engine in the loading phase. MATLAB/SIMULINK acts as a slave for AeroLink. Because of multi-platform, data transferring had to be done without affecting real time system response. AeroLink performs real time direct memory access to make data transfer with the MATLAB/SIMULINK model.

\section{RESULTS}

The new fuzzy logic control system is developed using the Fuzzy Logic Toolbox of MATLAB. Several scenarios are conducted on the new controller. Some of these scenarios are shown in Fig. 18 and Fig. 19. The demonstrated scenario in Fig. 18 is run with initial conditions of $2000 \mathrm{ft}$. $(609 \mathrm{~m}$.) altitude and $45 \mathrm{~m} / \mathrm{sec}$ speed. The controller performs good contour following to reach way point 2 (see Fig. 18(b)). Way point 2 is reached with error in azimuth equal -1.2 degrees and error in range equal to $450 \mathrm{~m}$. Way point 3 is reached with error in azimuth equal 3.2 degrees and error in range equal to $492 \mathrm{~m}$. Way point 4 is reached with error in azimuth equal 2.03 degrees and error in range equal to $403 \mathrm{~m}$. Way point 5 is reached with error in azimuth equal -1.8 degrees and error in range equal to $480 \mathrm{~m}$. Fig. 18(c) shows the $3 \mathrm{D}$ path for this scenario.

The second scenario, shown in Fig. 19, is run with initial conditions of $6000 \mathrm{ft}$. (1827 $\mathrm{m}$ ) altitude and $45 \mathrm{~m} / \mathrm{sec}$ speed. The controller performs good attack to reach way point 2 passing over both obstacles (which have altitudes of 1552, and 1167 respectively) (see Fig. 19(b)). Way point 2 is reached with error in azimuth equal 2.91 degrees and error in range equal to $424 \mathrm{~m}$. Way point 3 is reached after maneuver with an obstacle (which have altitude of $2334 \mathrm{~m}$ ) with error in azimuth equal 4.3 degrees and error in range equal to $403 \mathrm{~m}$. Way point 4 is reached with error in azimuth equal 3.9 degrees and error in range equal to $427 \mathrm{~m}$. Way point 5 is reached with error in azimuth equal 4.9 degrees and error in range equal to 413 m. Fig. 19(c) shows the 3D path for this scenario.

\section{CONCLUSION AND FUTURE WORK}

We have presented a new fuzzy logic control system for the navigation and obstacle avoidance of an Unmanned Aerial Vehicle. Initially, we use the Beaver mathematical model and we perform the required tests and verifications. This model is an openloop model under MATLAB/SIMULINK environment. Additionally, we develop a new fuzzy logic controller to perform navigation and obstacle avoidance. In order to verify the controller decisions, a new software simulation package "AeroLink" is developed using Microsoft Visual $\mathrm{C}++$. AeroLink is a multi-platform package that combines the mathematical power of MATLAB/SIMULINK and the real time graphics power of 
Visual $\mathrm{C}++$. AeroLink is developed to perform closed loop real time environment sensing. An effective methodology for the multi-platform real time data exchange is handled perfectly. Three LRFs are used for environment sensing. This work can be extended in the future by implementing it using FPGA technology, incorporating neural network with fuzzy logic and implementing this system with a real UAV.

\section{REFERENCES}

[1] Elizabeth Bone, Christopher Bolkcom, "Unmanned Aerial Vehicles: Background and Issues for Congress", (2003).

[2] Warren Williams, Michael Harris, "The Challenges of Flight-Testing Unmanned Air Vehicles", (2002).

[3] I. K Nikolos, L. Doitsidis, V. N. Christopoulos, N. Tsourveloudis, "Roll Control of Unmanned Aerial Vehicles using Fuzzy Logic", (2003).

[4] Y. Li, N. Sundararajan and P. Saratchandran, "Neuro - Controller Design for Nonlinear Fighter Aircraft Maneuver using Fully Tuned RBF Networks", Automatica, Vol. 37, pp. 1293-1301, (2001).

[5] C. Schumacher and S. N. Singh, "Nonlinear Control of Multiple U.A.Vs in Closecoupled Formation Flight", Proceedings of the AIAA Guidance, Navigation, and Control Conference, Denver CO, pp. 14-17, (2000).

[6] B. A. White, A. L. Blumel and E. J. Hughes, "A Robust Fuzzy Autopilot Design using Multi-criteria Optimization", International Journal of Fuzzy Systems, Vol.2, No.2, pp. 129-138, (2000).

[7] M. G. Perhinschi, "Parameter Optimization via Genetic Algorithm of Fuzzy Controller for Autonomous Air Vehicle", Proceedings of the AIAA Guidance, Navigation, and Control Conference, Portland OR, USA, pp. 790-797, (1999).

[8] FDC 1.2 - A Simulink Toolbox for Flight Dynamics and Control Analysis Marc

Rauw, 2nd edition, (2001), http://www.dutchroll.com

[9] Applications of Fuzzy Logic in Control Design, MATLAB® TECHNICAL COMPUTING BRIEF. 


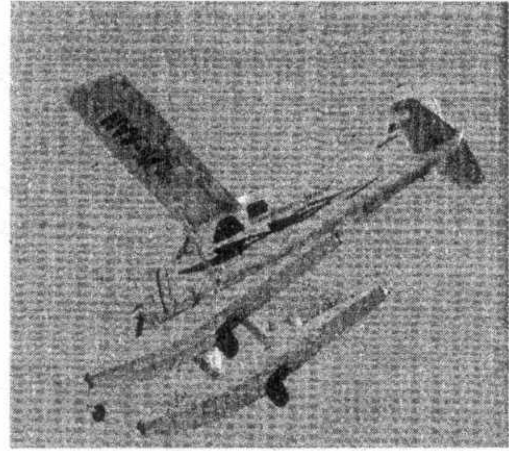

Fig. 1: DeHavilland DHC-2 'Beaver'
Table 1: Main technical characteristic of 'Beaver'

\begin{tabular}{|c|c|}
\hline Mass & $2288.231 \mathrm{Kg}$ \\
\hline Wing area & $23.23 \mathrm{~m}$ \\
\hline Wing span & $14.63 \mathrm{~m}$ \\
\hline $\begin{array}{c}\text { Mean aerodynamic } \\
\text { chord }\end{array}$ & $1.5857 \mathrm{~m}$ \\
\hline Fuselage length & $9.22 \mathrm{~m}$ \\
\hline Empty Weight & $14970 \mathrm{~N}$ \\
\hline Max. Power & $450 \mathrm{hp}$ at $2300 \mathrm{rpm}$ \\
\hline Operational Speed & $35-55 \mathrm{~km} / \mathrm{h}$ \\
\hline Max. altitude & $6000 \mathrm{ft}$ \\
\hline
\end{tabular}

Double-click
betore simulating:
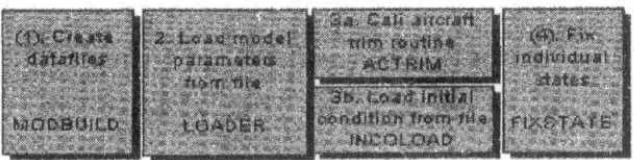

Double-click after
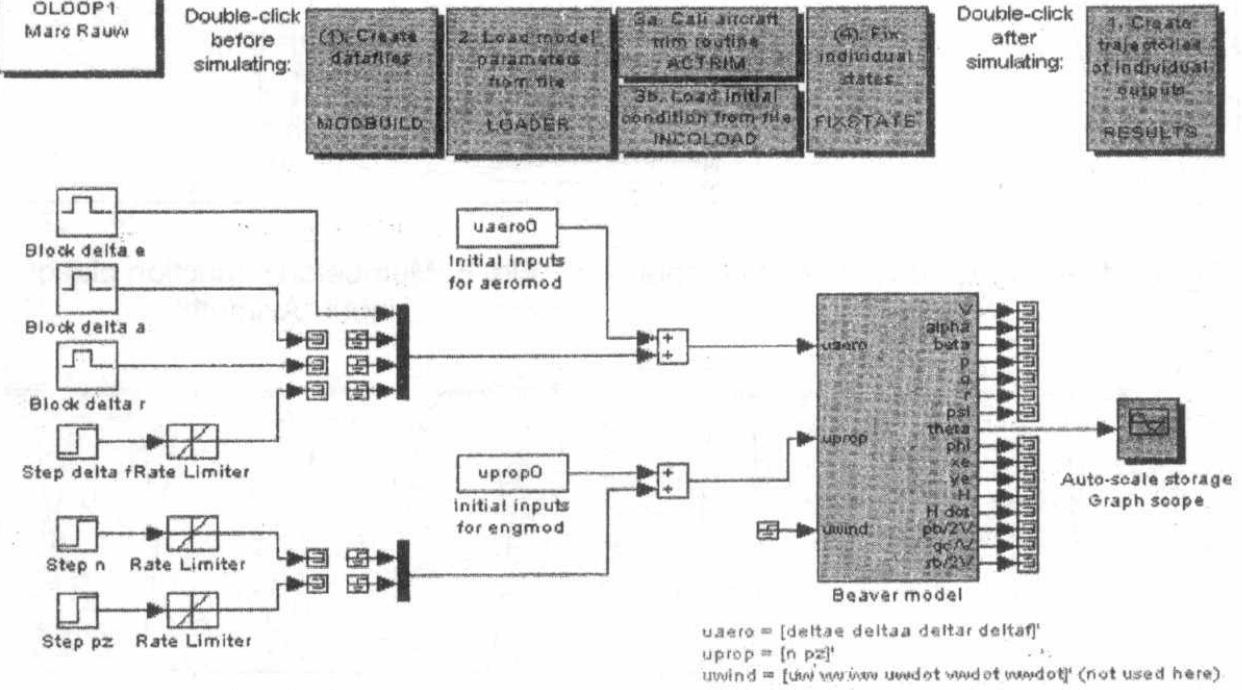

Fig. 2: Matlab/Simulink Beaver Model
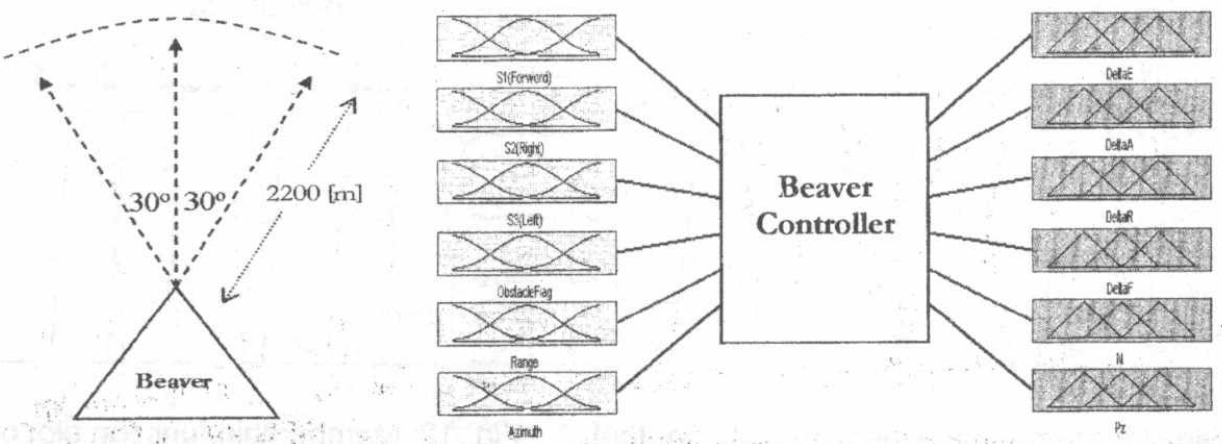

Fig. 3: sensors layout.

Fig. 4: New nav. \& obstacle avoidance Fuzzy controller 


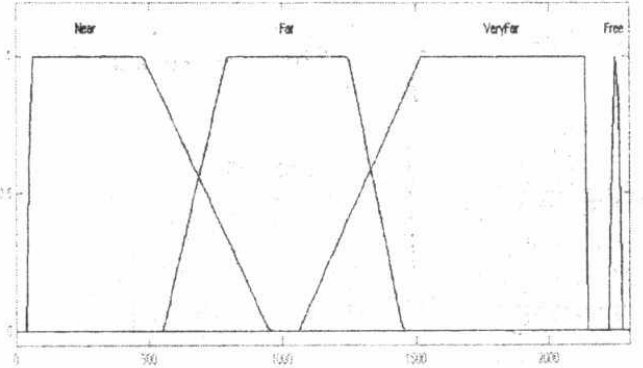

Fig. 5: Membership function plot of input "S1","S2" and "S3"

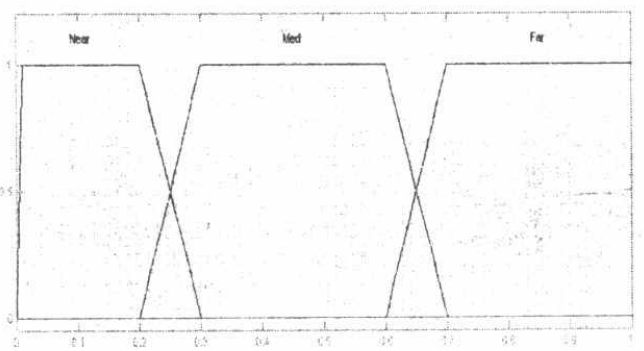

Fig. 7: Membership function plot of input "Range"

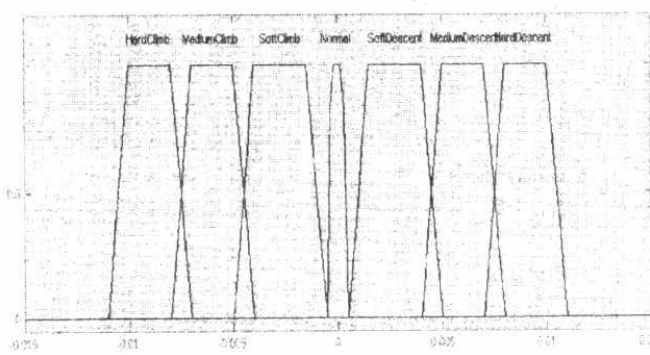

Fig. 9: Membership function plot of output " $\delta_{e} "$

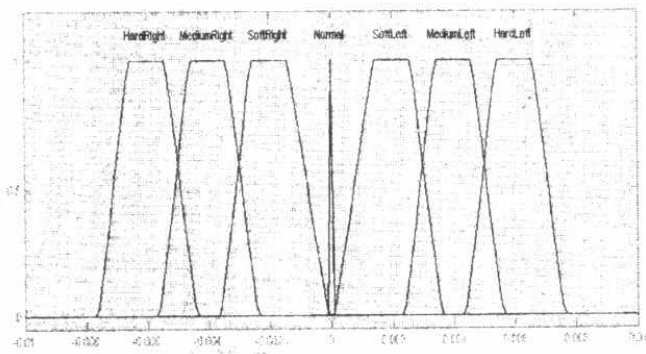

Fig. 11: Membership function plot of output " $\delta_{r} "$

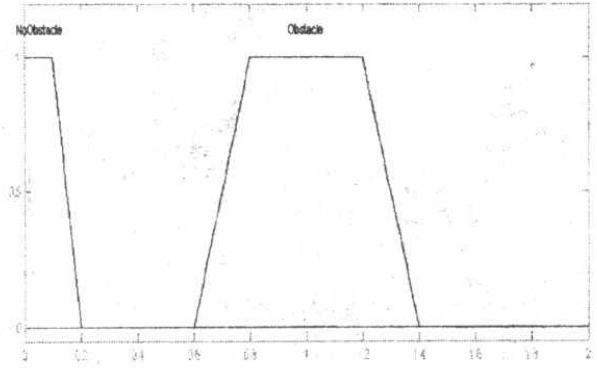

Fig. 6: Membership function plot of input "Obstacle Flag"

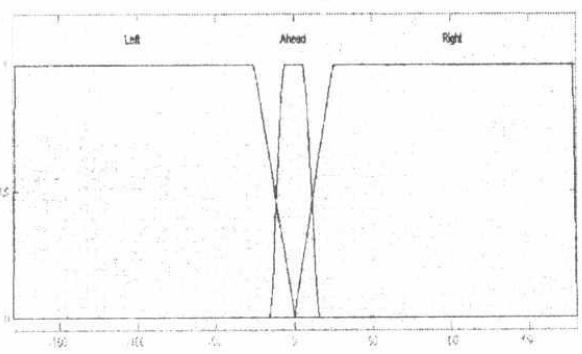

Fig. 8: Membership function plot of input "Azimuth"

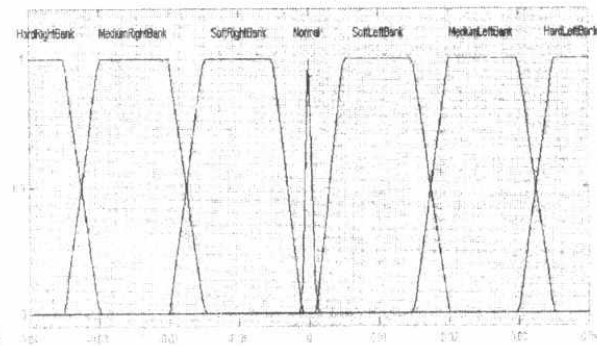

Fig. 10: Membership function plot of output " $\delta_{a}$ "

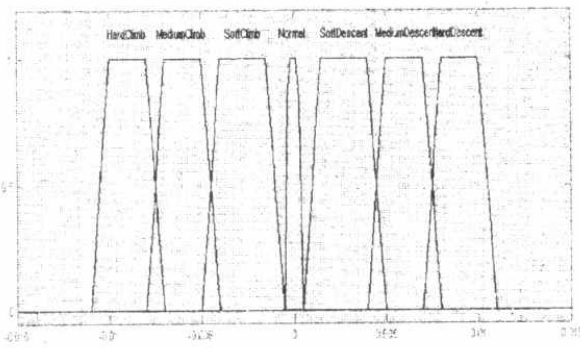

Fig. 12: Membership function plot of output " $\delta_{f}^{\prime \prime}$ 


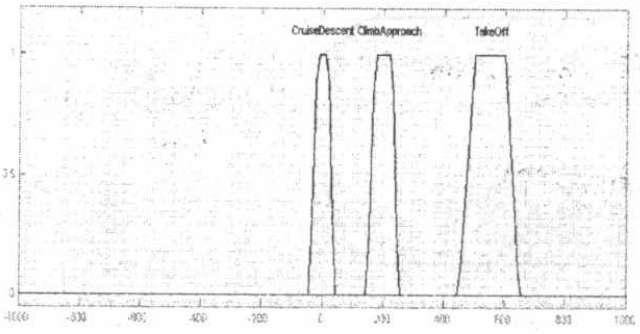

Fig. 13: Membership function plot of output "N"

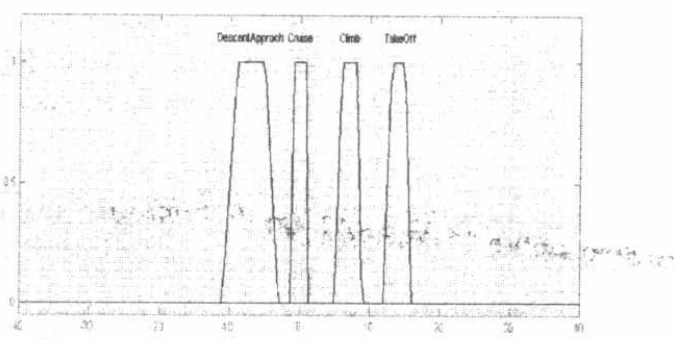

Fig. 14: Membership function plot of output "Pz"

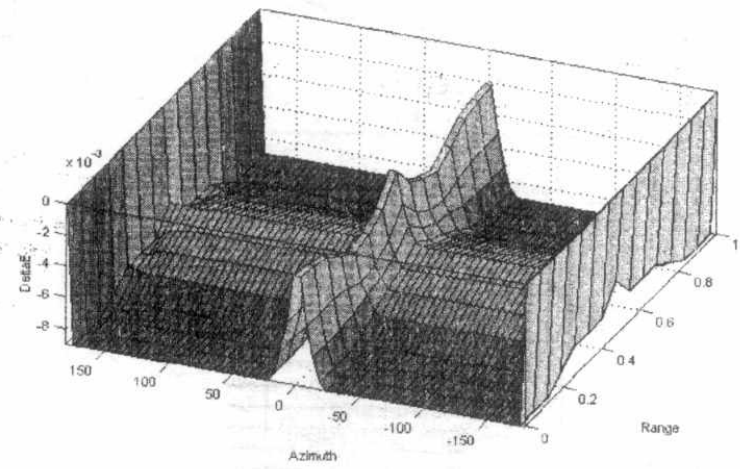

Fig. 15: The control surface

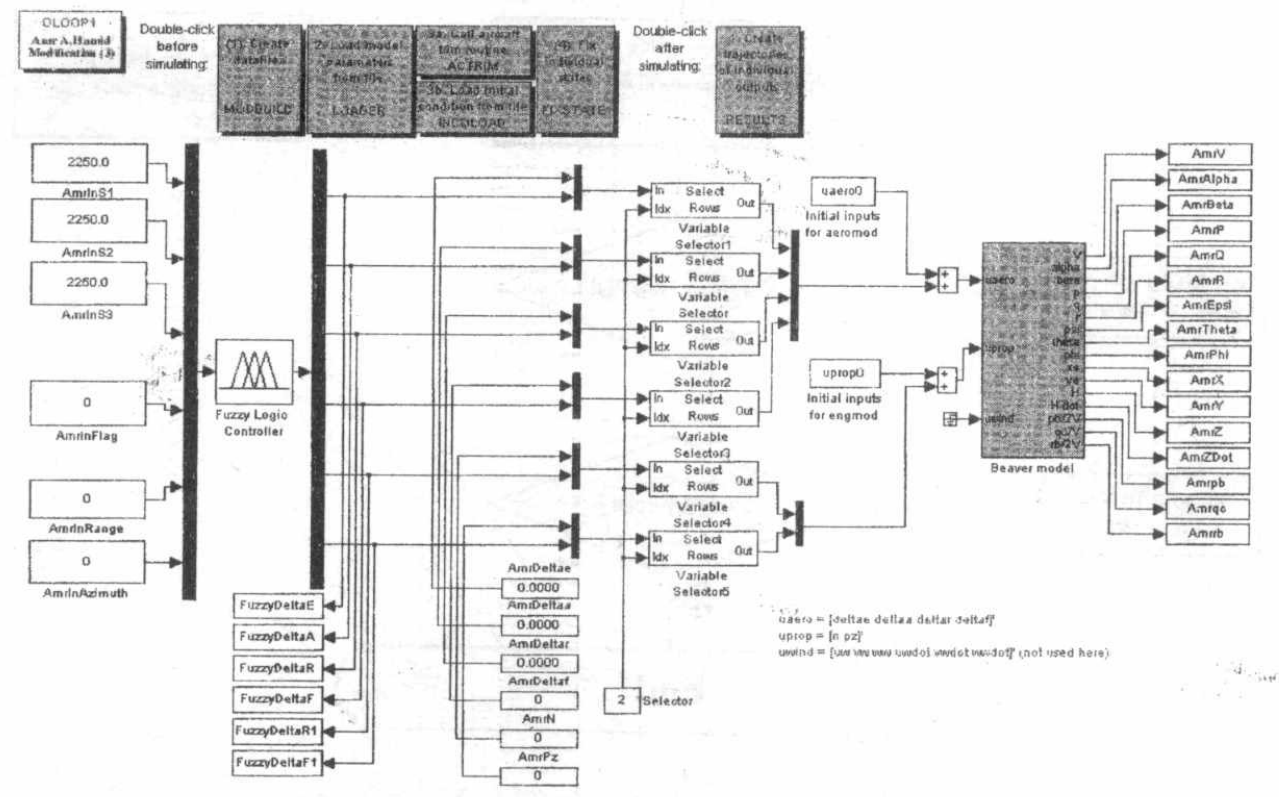

Fig. 16: Modified Beaver model for compatibility with the new controller 


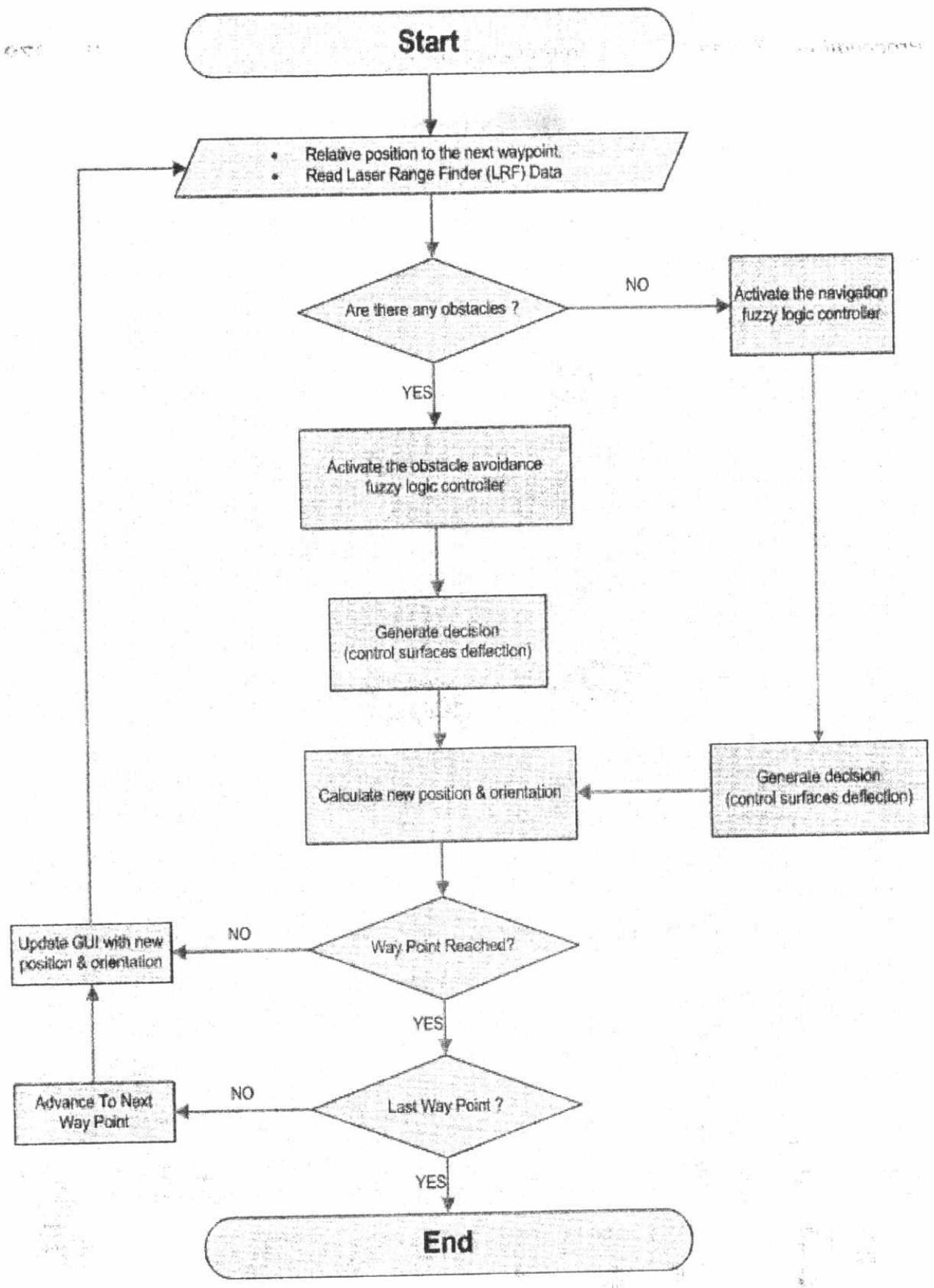

Fig. 17 : Sequence of operation flow chart 

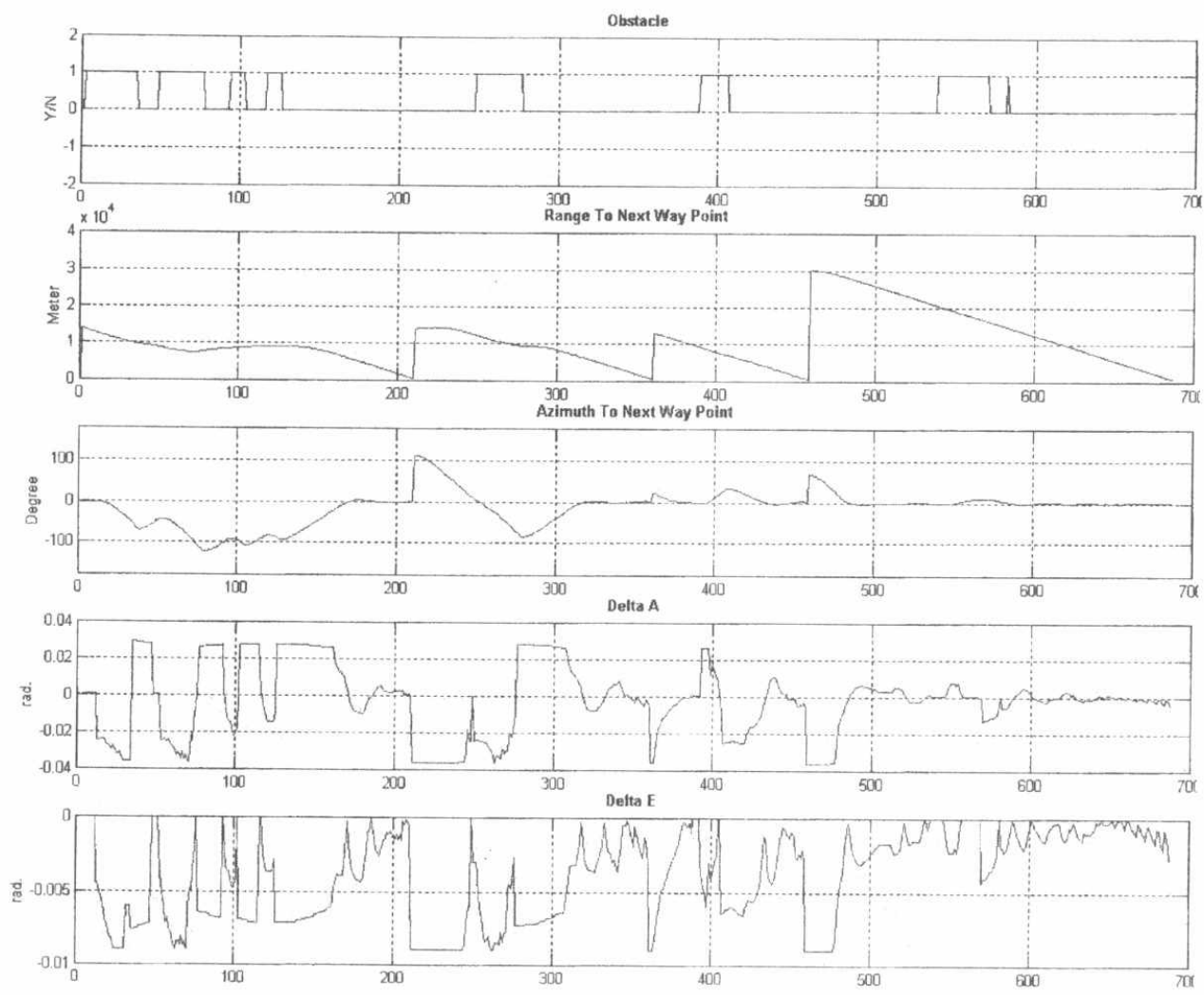

Fig. 18(a): Scenario parameters, obstacle status and controller actions

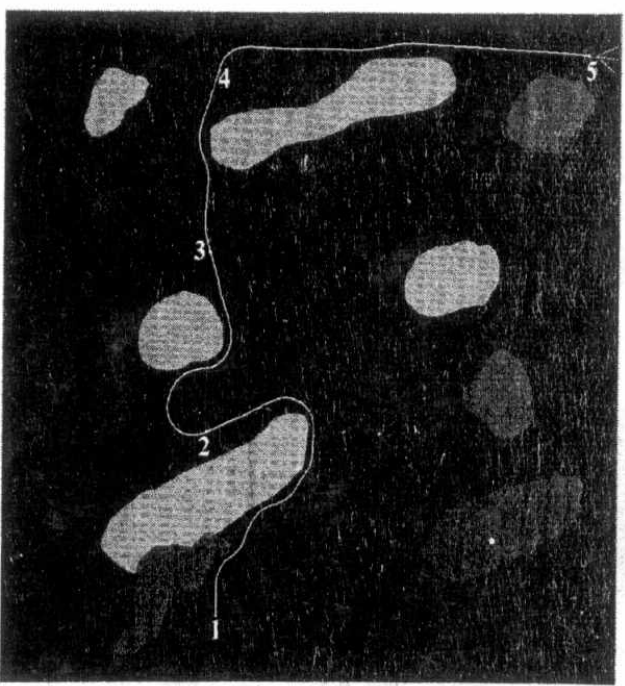

Fig. 18(b): 2D path for the flight

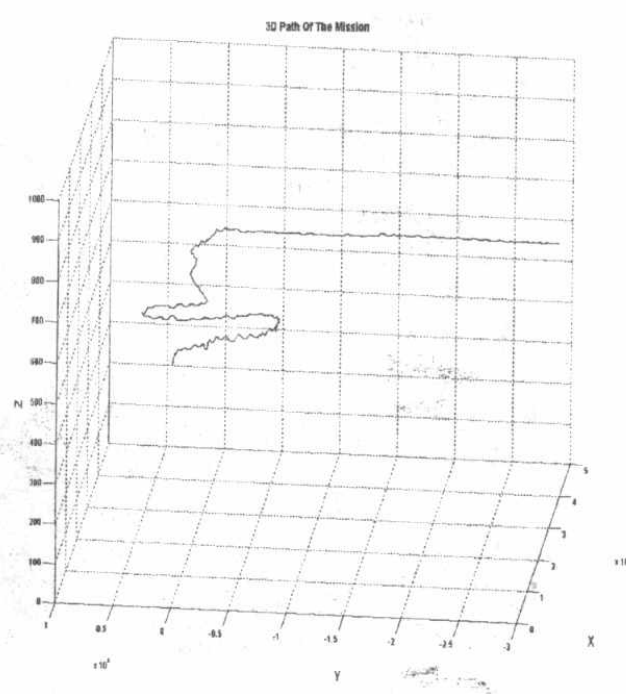

Fig. 18(c): 3D path for the flight 

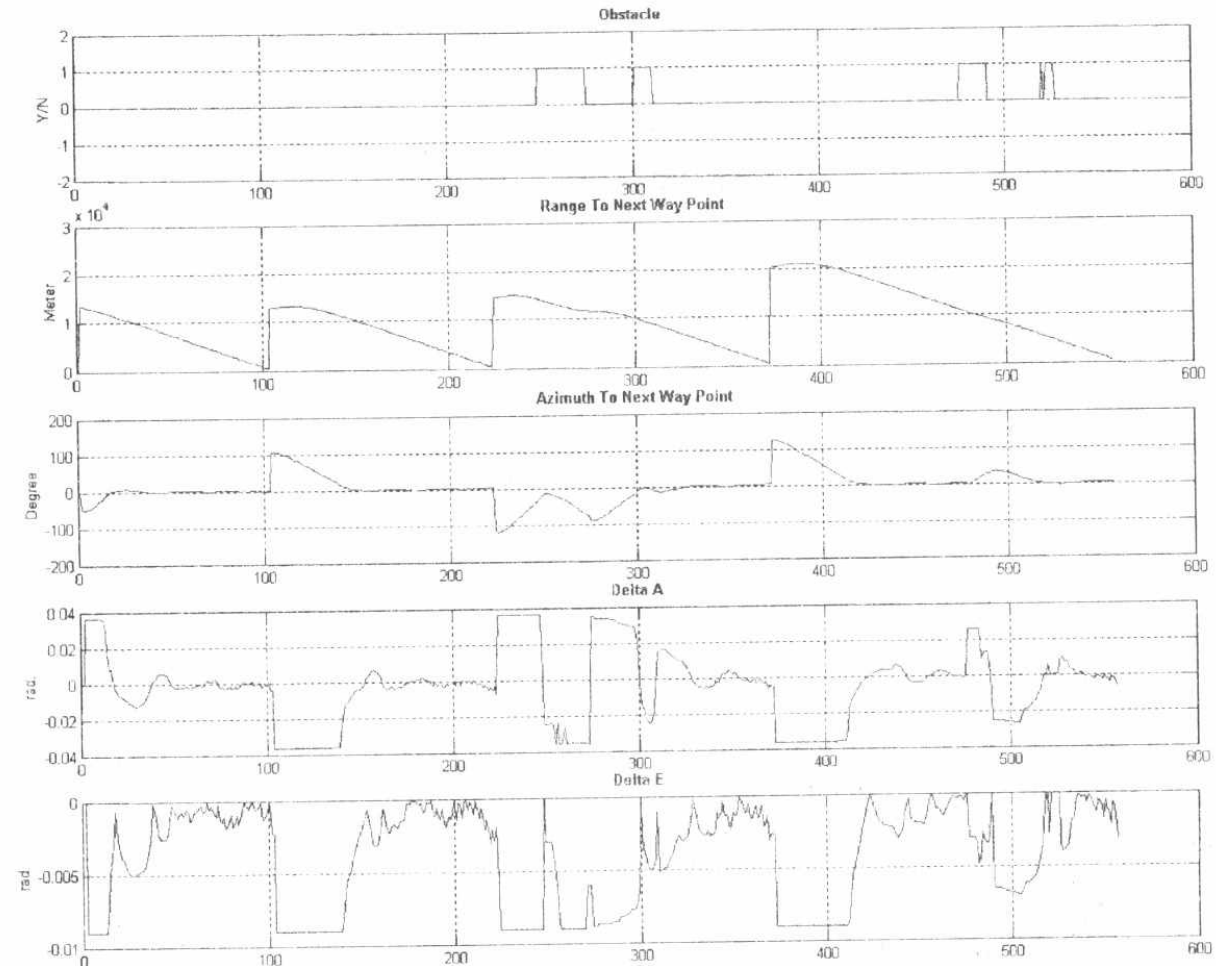

Fig. 19(a): Scenario parameters, obstacle status and controller actions

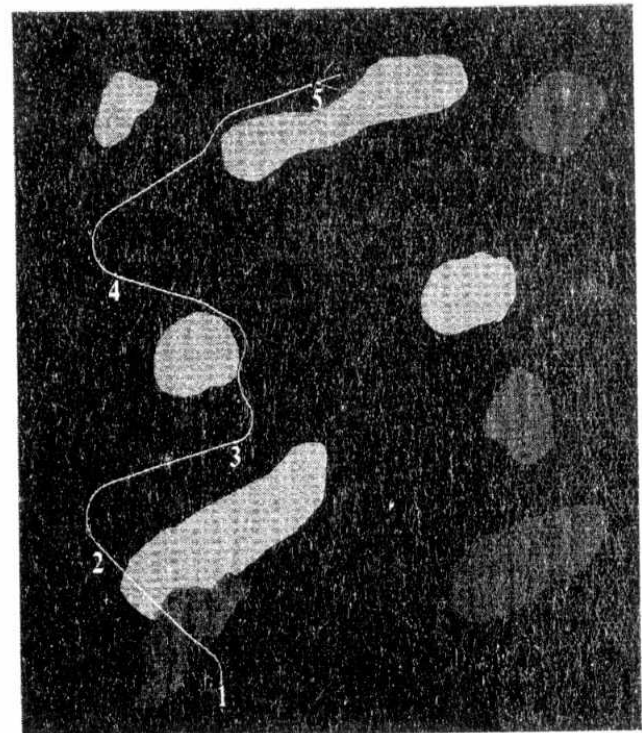

Fig. 19(b): 2D path for the flight

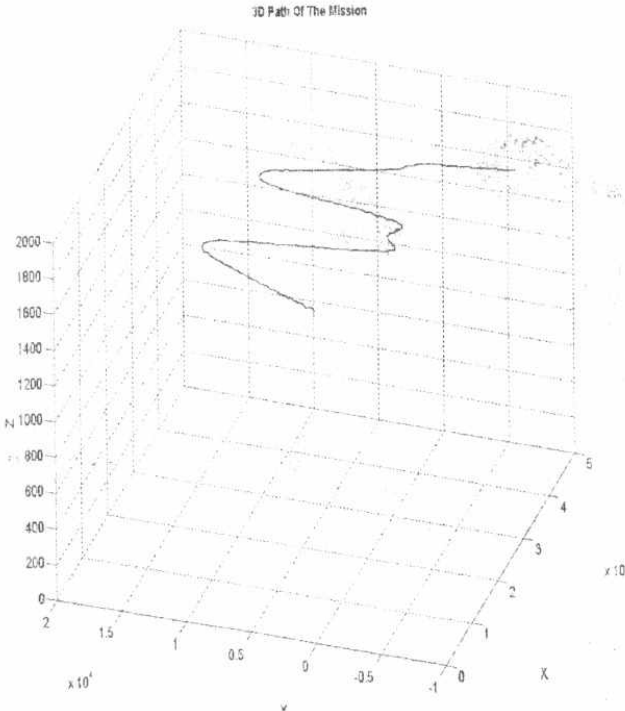

Fig. 19(c): 3D path for the flight 
RR-01 PERFORMANCE EVALUATION OF FREQUENCY AGILE RADAR IN PRESENCE OF CHIRP JAMMING

El-Mahdy, A. E, Abdel-latif Mohamed S., El-Gamel S. A.

RR-02 ASSESSMENT OF EARTH REMOTE SENSING MICROSATELLITE POWER SUBSYSTEM CAPABILITY DURING DETUMBLING AND NOMINAL MODES

$$
\text { M. Zahran, M. Okasha, Galina A. Ivanova }
$$

RR-03 A NEW APPROACH FOR MOVING TARGET DETECTION USING BARTLETT METHOD FOR SPECTRAL ESTIMATION

Abdel Rahman H. Elbardawiny, Khairy A. Elbarbary, Fathy M. Ahmed 